\title{
Muscle size explains low passive skeletal muscle force in heart failure patients
}

Fausto Antonio Panizzolo corresp., ${ }^{1,2}$ ， Andrew J Maiorana ${ }^{3,4}$ ， Louise H Naylor ${ }^{2}$ ， Lawrence G Dembo ${ }^{5}$, David G Lloyd $^{6}$ ， Daniel J Green ${ }^{2,7}$ ， Jonas Rubenson ${ }^{2,8}$

${ }^{1}$ John A. Paulson School of Engineering and Applied Sciences, Harvard University, Cambridge, MA, United States

2 The School of Sport Science, Exercise and Health, The University of Western Australia, Crawley, WA, Australia

3 Advanced Heart Failure and Cardiac Transplant Service, Royal Perth Hospital, Perth, WA, Australia

${ }^{4}$ School of Physiotherapy and Exercise Science, Curtin University, Perth, WA, Australia

5 Envision Medical Imaging, Perth, WA, Australia

${ }^{6}$ Centre for Musculoskeletal Research, Griffith Health Institute, Griffith University, Gold Coast, QLD, Australia

7 Research Institute for Sport and Exercise Science, Liverpool John Moores University, Liverpool, United Kingdom

8 Biomechanics Laboratory, Department of Kinesiology, The Pennsylvania State University, University Park, PA, United States

Corresponding Author: Fausto Antonio Panizzolo

Email address: fpanizzolo@seas.harvard.edu

Background. Alterations in skeletal muscle function and architecture have been linked to the compromised exercise capacity characterizing chronic heart failure (CHF). However, how passive skeletal muscle force is affected in $\mathrm{CHF}$ is not clear. Understanding passive force characteristics in CHF can help further elucidate the extent to which altered contractile properties and/or architecture might affect muscle and locomotor function. Therefore, the aim of this study was to investigate passive force in a single muscle for which non-invasive measures of muscle size and estimates of fiber force are possible, the soleus (SOL), both in CHF patients and age- and physical activity-matched control participants. Methods. Passive SOL muscle force and size were obtained by means of a novel approach combining experimental data (dynamometry, electromyography, ultrasound imaging) with a musculoskeletal model. Results. We found reduced passive SOL forces ( $\sim 30 \%$ ) (at the same relative levels of muscle stretch) in CHF vs. healthy individuals. This difference was eliminated when force was normalized by physiological cross sectional area, indicating that reduced force output may be most strongly associated with muscle size. Nevertheless, passive force was significantly higher in CHF at a given absolute muscle length (non length-normalized) and likely explained by the shorter muscle slack lengths and optimal muscle lengths measured in CHF compared to the control participants. This later factor may lead to altered performance of the SOL in functional tasks such gait. Discussion. These findings suggest introducing exercise rehabilitation targeting muscle hypertrophy and, specifically for the calf muscles, exercise that promotes muscle lengthening. 


\section{Muscle size explains low passive skeletal muscle force in heart}

2

3

4 Fausto A. Panizzolo ${ }^{1,2}$, Andrew J. Maiorana ${ }^{3,4}$, Louise H. Naylor ${ }^{1}$, Lawrence Dembo ${ }^{5}$, David G. Lloyd ${ }^{6}$, 5 Daniel J. Green ${ }^{1,7}$, and Jonas Rubenson ${ }^{1,8}$

6

$7{ }^{1}$ The School of Sport Science, Exercise and Health, The University of Western Australia, Crawley, WA, 8 Australia.

9 2John A. Paulson School of Engineering and Applied Sciences, Harvard University, Cambridge, MA, 10 United States.

$11{ }^{3}$ Advanced Heart Failure and Cardiac Transplant Service, Royal Perth Hospital, Perth, WA, Australia.

$12{ }^{4}$ School of Physiotherapy and Exercise Science, Curtin University, Perth, WA, Australia.

$13{ }^{5}$ Envision Medical Imaging, Perth, WA, Australia.

$14{ }^{6}$ Centre for Musculoskeletal Research, Griffith Health Institute, Griffith University, Gold Coast, QLD, 15 Australia.

$16{ }^{7}$ Research Institute for Sport and Exercise Science, Liverpool John Moores University, Liverpool, United 17 Kingdom.

${ }^{8}$ Biomechanics Laboratory, Department of Kinesiology, The Pennsylvania State University, University 19 Park, PA, United States.

20

21 Corresponding author: Dr. Fausto A. Panizzolo

22 John A. Paulson School of Engineering and Applied Sciences

23 Harvard University, 60 Oxford st, Cambridge, MA, USA. 


\section{ABSTRACT}

28 Background. Alterations in skeletal muscle function and architecture have been linked to the compromised exercise capacity characterizing chronic heart failure (CHF). However, how passive skeletal muscle force is affected in CHF is not clear. Understanding passive force characteristics in CHF can help further elucidate the extent to which altered contractile properties and/or architecture might affect muscle and locomotor function. Therefore, the aim of this study was to investigate passive force in a single muscle for which non-invasive measures of muscle size and estimates of fiber force are possible, the soleus (SOL), both in CHF patients and ageand physical activity-matched control participants.

Methods. Passive SOL muscle force and size were obtained by means of a novel approach combining experimental data (dynamometry, electromyography, ultrasound imaging) with a musculoskeletal model.

Results. We found reduced passive SOL forces $(\sim 30 \%)$ (at the same relative levels of muscle 40 stretch) in CHF vs. healthy individuals. This difference was eliminated when force was normalized by physiological cross sectional area, indicating that reduced force output may be most strongly associated with muscle size. Nevertheless, passive force was significantly higher in $\mathrm{CHF}$ at a given absolute muscle length (non length-normalized) and likely explained by the

44 shorter muscle slack lengths and optimal muscle lengths measured in CHF compared to the control participants. This later factor may lead to altered performance of the SOL in functional 46 tasks such gait. 
47 Discussion. These findings suggest introducing exercise rehabilitation targeting muscle 48 hypertrophy and, specifically for the calf muscles, exercise that promotes muscle lengthening. 49 


\section{INTRODUCTION}

52 Growing evidence suggests that architectural and functional deficiencies (e.g. strength) in the 53 skeletal muscle contribute to the limited ability to perform daily tasks and the overall poor 54 exercise tolerance that characterizes chronic heart failure (CHF) and to the progression of the 55 disease (Green et al., 2016). For example, it is apparent that patients with CHF have a reduction 56 in muscle size (Mancini et al., 1992; Minotti et al., 1993; Anker et al., 1999; Fülster et al., 2013) 57 and strength (as determined by net joint moments) in the lower limbs (Magnusson et al., 1994; Chua et al., 1995; Harrington et al., 1997; Sunnerhagen et al., 1998; Toth et al., 2006; Toth et al., 2010; Panizzolo et al., 2015) compared to healthy age-matched individuals; these reductions are also related to reductions in aerobic exercise capacity ( $\mathrm{V}_{2} \mathrm{O}_{2}$ peak) (Volterrani et al., 1994; Harrington et al., 1997; Panizzolo et al., 2015). It is still not clear, however, if the reduction in muscle function and aerobic capacity are associated primarily with reduced muscle size that is known to occur in CHF (Mancini et al., 1992; Fülster et al., 2013; Panizzolo et al., 2015) or if size-independent characteristics is an important determinant. Indeed, several studies that have measured both voluntary strength and muscle size in the quadriceps suggest that muscle size alone does not account for the loss of strength (Harrington et al., 1997; Toth et al., 2006; Toth et al., 2010). Resolving whether muscle size or other size-independent muscle properties are more closely linked to muscle function can prove important for guiding rehabilitation strategies in CHF.

To this extent, measurements of passive muscle forces and how they are related to muscle architecture can provide important information for understanding the mechanisms behind the

72 alterations in skeletal muscle function associated with CHF. In particular, they can shed further

73 light on whether skeletal muscle deficits at a whole muscle level are related primarily to 
74 reductions in muscle size without introducing variability arising from voluntary and/or twitch

75 contractions (Princivero et al., 2000; Oskouei et al., 2003). Passive forces are also functionally

76 relevant as they influence normal (Whittington et al., 2008) and pathological (Geertsen et al.,

77 2015) gait mechanics.

78 Our understanding of how passive skeletal muscle force is affected in CHF is currently

79 unclear. Passive forces in cardiac muscle are altered in CHF (Van der Velden, 2011), as well as

80 in diaphragm skeletal muscle (Van Hees et al., 2010). Surprisingly, as far as we are aware, only

81 one study (Van Hees et al., 2010) has investigated passive forces in appendicular skeletal muscle

82 in CHF and it has been conducted in a mouse model. This study reported unaltered passive

83 forces in the soleus (SOL) muscle of CHF-affected mice, compared to a control group, when

84 taking into consideration muscle size.

85 The SOL has been identified as a primary muscle in which tissue loss occurs in CHF

86 (Panizzolo et al., 2015; Green et al., 2016) and its size is strongly correlated with the reduced

87 exercise capacity present in CHF (Panizzolo et al., 2015) (more so than the gastrocnemius

88 synergist). The SOL is also functionally relevant as it has been identified as the main source of

89 mechanical work during gait (Mc Gowan et al., 2009). Furthermore, the SOL permits an

90 estimation of passive force in a single muscle (Rubenson et al., 2012; Tian et al., 2012) and thus

91 is a muscle of choice for muscle-specific analysis.

92 Therefore, the aim of this study was to investigate the passive forces in the SOL muscle

93 of CHF patients and age- and physical activity-matched control participants, including their

94 relationship to muscle architecture [physiological cross sectional area (PCSA), muscle length,

95 pennation angle]. We hypothesized that there would be a reduction in passive force in CHF

96 patients, compared to a healthy population. We further hypothesized that passive force would be 
97 similar after normalizing for the muscle PCSA, thus attributing alterations in passive force to 98 muscle size.

\section{MATERIALS AND METHODS}

\section{Participants}

102 Patients with CHF and age- and physical activity-matched control participants who were free

103 from other musculoskeletal disorders and lower limb musculoskeletal injuries were recruited for

104 this study. The CHF group included 12 participants ( 7 men, 5 women) in the class II-IV of the

105 New York Heart Association (NYHA) classification with an ejection fraction of 30.5 $\pm 9.6 \%$. (For

106 anthropometric characteristics and exclusion criteria see Table 1). The control group was

107 composed of 12 healthy participants recruited from the local community ( 8 men, 4 women). The

108 CHF group underwent regular exercise activity 2-3 times per week for $\sim 1$ hour per session

109 (treadmill walking and resistance weight training) as part of their standard patient care. The

110 control participants were selected from those reporting similar levels of weekly exercise,

111 assessed by means of a fitness questionnaire (Godin \& Shepard, 1985). All participants read and

112 signed an informed consent prior to participating in the study and all of the procedures were

113 approved by the Human Research Ethics Committee at The University of Western Australia

114 (approval ID: RA/4/1/2533) and Royal Perth Hospital (approval ID: 2011/019).

\section{Passive force estimates}

117 The procedures used to estimate passive SOL forces were similar to those adopted previously

118 (Rubenson et al., 2012), with the exception that passive force was measured during continuous

119 joint rotation. Passive moments were recorded with the participants sitting upright with their 
120 right foot and ankle positioned in a dynamometer (Biodex M3, Shirley, NY, USA) and with the

121 knee positioned at $120^{\circ}$ of flexion $\left(0^{\circ}\right.$ knee fully extended $)$ to mitigate the force contribution of

122 the gastrocnemius muscles (Maganaris, 2001). The net passive ankle joint moment $\left(M_{p}\right)$ was

123 computed by subtracting the moment generated by the Biodex rig and the weight of the foot

124 (Rubenson et al., 2012); the weight of the foot was expressed as a percentage of body mass. The

$125 M_{p}$ over a joint's range of motion passes through zero at an angle that approximates where

126 passive muscle forces reach zero (Silder et al., 2007) (Figure 1). Moment data recorded by the

127 dynamometer were filtered using $4^{\text {th }}$-order zero-lag $2 \mathrm{~Hz}$ low-pass Butterworth filter (MATLAB,

128 The MathWorks Inc., USA). To detect the inflexion point in $M_{p}$ where net dorsiflexion and

129 plantarflexion moment converge on zero we first fitted the joint angle $v s . M_{p}$ data with a $5^{\text {th }}$-order

130 polynomial and subsequently computed the first order derivative of this function (MATLAB,

131 The MathWorks Inc., USA) (Figure 1).

132 Contribution from synergist muscles are minimal at the joint postures adopted

133 (Maganaris, 2001; Silder et al. 2007; Rubenson et al., 2012). This was confirmed from OpenSim

134 musculoskeletal models simulations (Delp et al., 2007) further indicating that passive force from

135 synergist muscles were minimal at the recorded knee and ankle postures. The method described

136 above does not account for passive moments arising from joint articulations and skin, but these

137 are minimal compared to the passive moments arising from passive force in the Achilles tendon

138 (Costa et al., 2006).

139 Electromyography (EMG) from the tibialis anterior (TA), the medial and lateral 140 gastrocnemius muscles ( $\mathrm{MG}, \mathrm{LG}$, respectively) and the SOL were recorded during the trials

141 (Noraxon wireless system, Scotsdale, AZ, USA, $2000 \mathrm{~Hz}$ ) to ensure the muscles crossing the 142 ankle remained inactive. For each trial, real-time root-mean-square (RMS) waves of the muscles' 
143 activity were computed from the EMG signals (incorporating DC offset; Spike2 V7 software;

144 Cambridge Electronic Design, Cambridge, UK) (Rubenson et al., 2012). Soleus fascicle lengths

145 and pennation angle were recorded using dynamic B-mode ultrasound (Telemed, EchoBlaster

146 128, Lithuania; $25 \mathrm{~Hz}$ capture rate; $7.5 \mathrm{MHz} 60 \mathrm{~mm}$ linear array probe) following the placement

147 and image analysis procedures outlined previously (Rubenson et al., 2012; Panizzolo et al.,

148 2013). Simultaneous measurements of ankle joint flexion/extension angles were made using a

149 portable 3D motion capture system (Optitrack, Corvallis, Oregon, US, $100 \mathrm{~Hz}$ ). The net joint

150 moment, EMG, ultrasound images and joint angles were recorded synchronously (Micro1401-3;

151 Cambridge Electronic Design, Cambridge, UK; $2000 \mathrm{~Hz}$ ) as the ankle was cycled through its full

152 range of motion (the most plantarflexed and most dorsiflexed position tolerated by the

153 participant) at a constant speed of $5 \%$ s over three consecutive cycles.

154 Three initial warm-up cycles were performed prior the recording of any measurements.

155 The SOL passive force $\left(F_{p_{S O L}}\right)$ was computed continuously throughout the joint range of motion

156 as the joint underwent dorsiflexion. Passive force was calculated as per (Rubenson et al., 2012)

157 using the following equation:

158

$$
F_{p_{S O L}}=\frac{M_{p}}{r * \cos \theta}
$$

159 Where $r$ represents the Achilles moment arm data and $\theta$ the SOL pennation angle, measured 160 according to (Panizzolo et al., 2015).

161 Participant-specific Achilles moment arm data were established experimentally on a 162 separate testing day, following the method described previously in (Manal, Cowder \& 163 Buchanan, 2010). In this method B-mode ultrasound (Telemed, Echo Blaster 128, Lithuania) 164 was used to capture Achilles tendon images in the sagittal plane from the participants while their 165 foot was cycled passively at an angular velocity of $5 \%$ s across its range of motion in a Biodex 
166 dynamometer (M3, Biodex, Shirley, NY, USA). The ultrasound probe (7.5 MHz, $60 \mathrm{~mm}$ field of

167 view, linear array probe, $50 \mathrm{~Hz}$ capture rate) was placed longitudinally over the Achilles tendon,

168 so that the ankle joint was included in the field of view of the probe, using a stand-off gel pad

169 (Aquaflex, Parker, NJ, USA). Simultaneously, the trajectories of two retro-reflective markers

170 mounted on the ultrasound probe were recorded by means of a 3D motion capture system

171 (Optitrack, Corvallis, Oregon, US, $100 \mathrm{~Hz}$ ). Additional anatomical landmarks (first metatarsal,

172 calcaneus, medial malleoli and knee medial condyle) were tracked to calculate the ankle

173 flexion/extension joint angle. A 2D customized graphical interface was developed in Matlab to

174 display both the ultrasound images and the ultrasound probe and the medial malleolus markers in

175 the same coordinate system. The line of action of the Achilles tendon was digitized in this

176 common coordinate system and the moment arm was computed as the perpendicular distance

177 between the midline of action of the tendon and the medial malleolus, which was used as an

178 estimate of the ankle joint center. This procedure was performed at 10 ankle joint angles that

179 spanned the joint's range of motion. A 10-point moment arm-joint angle curve was obtained for

180 each participant by using a polynomial fit of the moment arm-joint angle data.

181 We defined the fascicle slack length $\left(L_{\text {slack }}\right)$ as the length where passive SOL forces are

182 first generated. $L_{\text {slack }}$ was estimated as the point where the net passive dorsiflexion and

183 plantarflexion moments converge on zero (Figure 1). The fascicle length at the maximum

184 tolerated dorsiflexion angle was defined as the maximal fascicle length $\left(L_{\max }\right)$. Absolute and

185 normalized passive SOL force-length (F-L) curves were established for each participant.

186 Absolute passive F-L curves used the measured $F_{p_{S O L}}$ in Newtons and fascicle lengths $(L)$ in $\mathrm{mm}$.

187 Normalized passive F-L curves were created from absolute passive F-L curves by dividing each

188 participant $F_{p_{S O L}}$ by their SOL PCSA and by dividing $L$ by $L_{\text {slack }}$ (this normalized length is 
189 referred as $L_{\text {norm }}$ from hereinafter). The PCSA was determined from underwater 3D ultrasound

190 scans which allowed to obtain information relative to SOL muscle volume (Telemed,

191 EchoBlaster 128, Lithuania; Stradwin, Medical Imaging Research Group, Cambridge University

192 Engineering Department, UK) following (Panizzolo et al., 2015). To enable the comparison of

193 absolute $F_{p_{S O L}}$ between groups, $F_{p_{S O L}}$ was determined at a percent fascicle stretch of $0 \%, 20 \%$,

$19440 \%, 60 \%, 80 \%$ and $100 \%$ of the maximum fascicle stretch, where percent fascicle stretch was

195 defined as $\left(\left(L-L_{\text {slack }}\right) \div\left(L_{\text {max }}-L_{\text {slack }}\right)\right) * 100$. The same procedure was done to compare passive

196 moment data over both angle and muscle length ranges. Passive fascicle stiffness was computed

197 for each participant as the slope of the absolute F-L curves between $L_{\text {slack }}$ and $40 \%$ stretch $\left(k_{1}\right)$

198 and between $60 \%$ - 100\% stretch $\left(k_{2}\right)$. In order to compare the normalized passive F-L curves we

199 evaluated the normalized $F_{p_{S O L}}$ at a set of $L_{n o r m}$ between 1.0 and 1.4 (i.e. strain of $0-40 \%$ ) using

200 intervals of 0.05 . A peak $L_{n o r m}$ was set to 1.4 as this represented the average maximum $L_{n o r m}$ that

201 the participants achieved at their end range of ankle dorsiflexion. The normalized $F_{p_{S O L}}$ was

202 computed for each individual for the interval described above by fitting the normalized $F_{p_{S O L}}$ and

$203 L_{n o r m}$ data using a $1^{\text {st }}$-order exponential equation (Gollapudi \& Lin, 2009) with subject-specific

204 constants. In some circumstances where the set range exceeded the experimental $L_{\text {norm }}$ the

205 normalized $F_{p_{S O L}}$ values were extrapolated from the exponential equation. Stiffness was

206 computed between $L_{\text {norm }}$ of 1.0 and $1.2\left(k_{1 \text { norm }}\right)$ and 1.2 and $1.4\left(k_{2 \text { norm }}\right)$.

\section{Active forces estimates}

As an ancillary comparison of the muscle lengths, we also analyzed peak active muscle

210 forces at different ankle angles (and thus muscle lengths) to generate an active force-length 
211 relationship. It has previously been shown experimentally, both in the human SOL muscle

212 (Rubenson et al., 2012) and in non-human muscle (Azizi \& Roberts, 2010) that optimal muscle

213 lengths $\left(L_{0}\right.$; lengths where peak active isometric forces are generated) correspond closely with

$214 L_{\text {slack }}$ Because of the importance of $L_{\text {slack }}$ in our analyses of length-dependent passive muscle

215 force and muscle stiffness we chose to also assess $L_{0}$ as an additional test for differences in

216 fascicle lengths between groups. The main purpose of performing the active force-length curve

217 for the SOL muscle was thus to improve our assessment of length-dependent passive force and

218 muscle stiffness that relies on length normalization, rather than insights into active force 219 production per se.

220 The protocol used in this study to obtain predictions of moments and force generated by

221 the SOL (as well as the moments and force generated by synergist muscles and by the co-

222 contraction of dorsiflexor muscles) expands on the procedures established in (Rubenson et al.,

223 2012). It uses a combination of experimental net moment measurements from dynamometry,

224 ultrasound fascicle imaging, electromyography and a scaled participant-specific musculoskeletal

225 model in OpenSim 2.0.2 (Delp et al., 2007). Predictions were performed with the knee in a

226 flexed position $\left(>120^{\circ}\right)$ and over a range of ankle angles from $\sim-20^{\circ}$ dorsiflexion to $30^{\circ}$

227 plantarflexion (the ankle range of motion varied between individuals ranging from individual

228 maximum dorsiflexion of $-30^{\circ}$ to individual maximal plantarflexion of nearly $50^{\circ}$ ). The muscle

229 length that corresponded with the maximal peak active force was designated as $L_{0}$.

230 First, a generic OpenSim lower-limb model (Arnold et al., 2010) was scaled using each

231 participant's joint axes and centers determined via motion capture data (8-camera VICON MX

232 motion capture system, Oxford Metrics, UK; $100 \mathrm{~Hz}$ ) from participants in a standing posture as

233 well as dynamic joint motions (Besier et al., 2003). From these trials, an inverse kinematics 
234 algorithm was run on the position of 26 retroreflective spherical markers placed on anatomical

235 landmarks and on functionally determined joint centers (Besier et al., 2003), that minimized the

236 distance between the OpenSim model markers and the retroreflective and the functionally

237 determined markers. These participants-specific models were positioned to match the

238 participant's optically recorded ankle and knee joint posture and in turn used to predict $M_{\text {dorsi }}$ and $239 M_{\text {Syn }}$ (see below).

240 The moment generated by the plantarflexors $\left(M_{\text {plant }}\right)$ during the maximal voluntary 241 isometric plantarflexion contractions $\left(M V C_{\text {plant }}\right)$ was calculated as:

$$
M_{\text {plant }}=M_{\text {peak }}-\Delta M_{p}+M_{\text {dorsi }}
$$

243 where $M_{\text {peak }}$ is the peak net ankle joint moment (calculated as the difference between the Biodex

244 recorded moment during $M V C_{\text {plant }}$ and the moment at rest), $\Delta M_{p}$ represents the difference in the

245 estimated passive SOL moment during the $M V C_{\text {plant }}$ and the passive SOL moment at rest prior to

246 the contraction, and $M_{\text {dorsi }}$ is the moment generated by the co-contraction of the dorsiflexors

247 muscles.

$248 \Delta M_{p}$ was calculated as:

$$
\Delta M_{p}=\left(F_{p_{S O L}}^{\text {contr }} * \cos \theta^{\text {contr }} * r^{\text {contr }}\right)-\left(F_{p_{S O L}}^{\text {rest }} * \cos \theta^{\text {rest }} * r^{\text {rest }}\right)
$$

where $F_{p_{S O L}}$ was obtained for both the fascicle length at the $M V C_{\text {plant }}$ and the fascicle length during the rest period just prior to contraction using a linear interpolation of the passive F-L

252 relationship (rest and contr superscripts designate rest or $M V C_{\text {plant }}$, respectively). $r^{\text {contr }}$ was

253 estimated by increasing the value predicted from the experimental Achilles moment arm- joint

254 angle equation (described above) by $20 \%$ to take in account the increase in moment arm distance 255 reported during $M V C_{\text {plant }}$ with respect to length at rest (Maganaris et al., 1998). 
maximal isometric forces of all the dorsiflexors (tibialis anterior, extensor digitorum longus, extensor hallucis longus, peroneus tertius) were adjusted by the same percentage increase or 259 decrease so that the predicted model's peak isometric dorsiflexion moment at $100 \%$ activation ( $\left.260 M V C_{d o r s i}\right)$ matched that of the participant's experimental maximum $M_{\text {dorsi }}$ recorded in the Biodex 261 dynamometer at $10^{\circ}$ plantarflexion, the angle that corresponds approximately to optimal 262 dorsiflexion moments (Silder et al., 2007). The $M V C_{\text {dorsi }}$ were performed only at this joint angle 263 to reduce the total numbers of contractions performed and time spent in the experimental 264 protocol by each participant. This was an important consideration because of the general high 265 fatigability of CHF patients. In this procedure, the OpenSim model was positioned to match the 266 participant's optically recorded ankle and knee joint posture. In subsequent measurements of $267 M V C_{\text {plant }}$ the $M_{\text {dorsi }}$ was predicted by the OpenSim model by prescribing an activation to all of the 268 dorsiflexors equal to the ratio of the TA's peak EMG (linear envelope) during the $M V C_{\text {plant }}$ to its 269 peak EMG (linear envelope) from the $M V C_{d o r s i}$ trial; i.e. this assumed the same activation level 270 for all dorsiflexors.

271 To take into account the contribution of synergist muscles we predicted the relative 272 percentage contribution of each plantarflexors muscle to the total plantarflexor moment in 273 OpenSim $\left(M_{S y n}\right)$ by prescribing the recorded ankle and knee angles and $100 \%$ activation of all 274 plantarflexor muscles (peroneus longus, peroneus brevis, flexor hallucis, tibialis posterior, flexor 275 digitorum, MG, LG and SOL). The percent contribution of the OpenSim SOL to the total 276 predicted moment was applied to the experimental $M V C_{\text {plant }}$ to define the moment generated by 277 the participant's SOL $\left(M_{a_{S O L}}\right)$. Lastly, peak voluntary active SOL force production $\left(F_{a_{S O L}}\right)$ was 278 calculated as: 


$$
F_{a_{S O L}}=\frac{M_{a_{S O L}}}{r^{\text {contr }} * \cos \theta^{\text {contr }}}
$$

These active force trials were performed only by the participants that were able to tolerate

281 a prolonged protocol ( $\mathrm{n}=7$ and $\mathrm{n}=8$, for control and CHF participants, respectively).

\section{Statistical analysis}

284 Differences in the absolute (non-normalized) passive moment-angle, moment-length and F-L 285 curves were assessed by testing if $F_{p_{S O L}}$ were different between groups (CHF and control), and if 286 joint angles at which the passive forces occurred and/or fascicle lengths were affected in the $287 \mathrm{CHF}$ group, by using a two-way (CHF/control) repeated measures $(0 \%, 20 \%, 40 \%, 60 \%, 80 \%$ 288 and $100 \%$ of angular excursion or muscle stretch, respectively) ANOVA, with Bonferroni post 289 hoc tests. Similar two-way repeated measures ANOVAs were also performed on the normalized 290 F-L curves using the $L_{\text {norm }}$ set range $(1.0$ - 1.4). A two-tailed unpaired Student's t-test with 291 significance level of $\mathrm{p}<0.05$ was used to determine significant differences in the $L_{\text {slack }} L_{\text {max }}$, the 292 maximal fascicle stretch, and $L_{0}$, as well as in the passive fascicle stiffness $\left(k_{1}, k_{2}, k_{1 \text { norm }}\right.$ and $\left.293 k_{2 \text { norm }}\right)$ and in the PCSA between the groups. Finally, we performed a linear regression analyses 294 between peak $F_{p_{S O L}}$ at $L_{\text {norm }}$ of 1.4 and PCSA and between muscle volume across groups. 295 Statistical analysis was performed in SPSS (IBM, Statistics 21, USA).

\section{RESULTS}

298 No main effect of group was found in the joint angle between the CHF and control groups $(\mathrm{p}=$ 299 0.42) (Figure 2a). A main effect of group on net passive ankle joint moment was found $(\mathrm{p}=$ 300 0.01) with lower passive moment in the CHF group compared to the control group at relative 
301 levels of angular excursion and fascicle stretch, although no statistically significant interaction

302 effect was found $(\mathrm{p}=0.40)$ between group and joint angle (Figure $2 \mathrm{a}-\mathrm{b})$.

303 A main effect of group on absolute $F_{p_{S O L}}(\mathrm{~N})$ was found $(\mathrm{p}=0.03)$ with lower absolute

$304 F_{p_{S O L}}$ in the CHF group compared to the control group at relative levels of fascicle stretch,

305 although no statistically significant interaction effect was found $(p=0.11)$ between group and

306 level of stretch. No differences were found in $k_{1}$ and $k_{2}$ between the groups $(\mathrm{p}=0.32 ; \mathrm{ES}=0.51$

307 and $\mathrm{p}=0.85 ; \mathrm{ES}=0.09)($ Figure $3 \mathrm{a})$, with stiffness exhibiting high variability. The $L_{\max }$ was

308 significantly shorter in the CHF group compared to the control group $(\mathrm{p}=0.046 ; \mathrm{ES}=0.96)$,

309 although no statistically significant differences were found in $L_{\text {slack }}(\mathrm{p}=0.11 ; \mathrm{ES}=0.76)$ and in

310 the maximal fascicle stretch $\left(L_{\text {max }}-L_{\text {slack }}\right)(\mathrm{p}=0.34 ; \mathrm{ES}=0.44)($ Table 2$)$ or maximal fascicle

311 strain $(p=0.70 ; E S=0.09)$.

312 A significantly smaller SOL PCSA was found in the CHF with respect to the control

313 group $(\mathrm{p}=0.02 ; \mathrm{ES}=1.25)($ Table 2$)$. No main effect was found in the PCSA-normalized $F_{p_{S O L}}$

$314\left(\mathrm{~N} \mathrm{~cm}^{-2}\right)$ between the CHF and control groups when using the $L_{n o r m}$ strain range of 1.0-1.4 $(\mathrm{p}=$

315 0.46) (Figure $3 b$ ), nor was there an interaction effect between the PCSA-normalized $F_{p_{S O L}}$ and

316 normalized lengths $(\mathrm{p}=0.52)$. Normalized passive fascicle stiffness $\left(k_{1 \text { norm }}\right.$ and $\left.k_{2 \text { norm }}\right)$ were

317 likewise variable and also not significantly different between the groups $(\mathrm{p}=0.42 ; \mathrm{ES}=0.44$ and

$318 \mathrm{p}=0.54 ; \mathrm{ES}=0.33)($ Figure $3 \mathrm{~b})$.

$319 L_{0}$ determined from the active force-length data was significantly shorter $(\sim 22 \%)$ in the

320 CHF group compared to the control group $(\mathrm{p}=0.04 ; \mathrm{ES}=0.96)($ Table 2$)$. The maximal $F_{a_{S O L}}$

321 and corresponding $L_{0}$ occurred at approximately $10^{\circ}$ dorsiflexion. The $F_{a_{S O L}}$ at both shorter and

322 longer fascicle lengths relative to $L_{0}$ decreased, characteristic of the muscle force-length 
323 relationship (Figure 4). $L_{0}$ was not significantly different from $L_{\text {slack }}$ in either the control or CHF

324 groups $(\mathrm{p}=0.33$ and $\mathrm{p}=0.39$, respectively; Table 2$)$.

325 A significant correlation was found between peak $F_{p_{S O L}}$ at $L_{\text {norm }}$ of 1.4 and muscle volume

$326(\mathrm{p}<0.01 ; \mathrm{r}=0.76)$ while no significant correlation was reported between peak $F_{p_{S O L}}$ at $L_{\text {norm }}$ of

3271.4 and PCSA $(\mathrm{p}=0.06 ; \mathrm{r}=0.46)$.

328

329 DISCUSSION

330 The present study provides, to the best of our knowledge, the first estimate of in vivo passive

331 human skeletal muscle force-length properties in CHF. As predicted, higher absolute $M_{p}$ and

$332 F_{p S O L}$ were produced in the control group for a given amount of muscle stretch (Figure 2, 3).

333 However, and also in agreement with our hypothesis, passive force was not different after

334 normalizing by muscle PCSA, nor is passive muscle stiffness affected. These results indicate that

335 muscle size rather than intrinsic muscle properties is a major factor influencing passive forces in

336 CHF SOL muscle. This was further supported by the correlation between peak $F_{p_{S O L}}$ and muscle

337 size across both the CHF and control groups. This finding stands in contrast to previous work

338 reporting stiffer cardiac muscle due to alterations in the titin structure $(W u, 2002)$ or decreased

339 passive force of the diaphragm, due to titin loss (Van Hees et al., 2010) in CHF. On the other

340 hand, our results do corroborate data from passive skeletal muscle properties in the mouse SOL,

341 in which passive forces from CHF-affected animals were likewise not altered after normalizing

342 to muscle cross sectional area (Van Hees et al., 2010).

343 It was surprising, however, that for a given absolute muscle length, passive force was

344 significantly higher in CHF SOL compared to the control group. This unexpected finding stems

345 from the fact that over the same ankle range of motion the passive muscle lengths are shorter in 
346 CHF patients, in particular at maximal stretch (Figure 2, 3). The result is that for the same

347 absolute muscle length (above $L_{\text {slack}}$ ) the CHF muscle has undergone greater strain, thus

348 generating greater force in titin and other passive load bearing muscle components. Previous

349 experimental studies (Azizi \& Roberts, 2010; Winters et al., 2011; Rubenson et al., 2012) have

350 shown agreement between the onset of passive force generation $\left(L_{\text {slack }}\right)$ and $L_{0}$ (optimal length for

351 active force production). The estimate of $L_{0}$ in the present study was similar to $L_{\text {slack }}$ for both

352 groups but significantly $(\mathrm{p}<0.05)$ shorter in the CHF group (Table 2$)$. The shorter $L_{\text {slack }}$ and $L_{0}$ in

353 CHF patients may indicate that the SOL has undergone a loss of in-series sarcomere numbers, a

354 contributing factor to the reduced muscle size (Panizzolo et al., 2015). It was also surprising that,

355 despite their shorter muscle fascicles, CHF patients underwent the same ankle range of motion

356 and a similar SOL muscle strain across this range of motion (Figure 2, Table 2). Therefore, the

357 'effective' stiffness of the muscle, the amount of force resulting at the maximal stretch of the

358 muscle (as indicated by $k_{1}$ and $k_{2}$ ), are similar between groups despite the passive force at the

359 same absolute muscle length being substantially greater in CHF patients. The passive moments at

360 equivalent ankle angle excursions (indicative of the ankle's effective angular stiffness) are

361 likewise similar between $\mathrm{CHF}$ and control groups (Figure 2). This is true except for a moderately

362 higher moment, and absolute force, in the control group at the participant's peak dorsiflexion

363 angle (Figure 2, 3) although these angles are rarely achieved during normal movement tasks. The

364 Achilles moment arms were similar between the control and CHF group suggesting that greater

365 Achilles strain might explain the similarity in joint and muscle excursions. This is partially

366 supported by the smaller tendon cross sectional area reported in CHF (Panizzolo et al., 2015).

368 Functional implications 
369 Our results are consistent with the observation that muscle size dictates functional deficits in

370 CHF (Magnusson et al., 1994). Exercise that promotes hypertrophy should therefore be a focus

371 for restoring functional capacity in leg muscles. Exercise prescription for CHF is becoming

372 commonplace, but programs that include specifically designed lower limb resistance training

373 might be especially promising (Maiorana et al., 2000).

374 Our results also offer insight into the gait mechanics of CHF patients (Panizzolo et al.,

375 2014). The combination of the shorter SOL muscle fascicles in CHF patients and their greater

376 dorsiflexion during mid-stance of gait (Panizzolo et al., 2014) may cause significantly greater

377 SOL strain. This might lead to the muscle operating on to the descending limb of the F-L curve

378 where large passive forces develop (Rassier, MacIntosh \& Herzog., 1999; Rubenson et al.,

379 2012). In this scenario CHF patients would rely more on their passive forces to support the

380 plantarflexion moment during walking, which has the benefit of reducing metabolically

381 expensive active force development. This may help explain why CHF patients rely

382 proportionately more on their ankle for powering walking as speed and metabolic demand

383 increases (Panizzolo et al., 2014). However, whilst metabolically advantageous, this mechanism

384 might lead to greater lengthening-induced muscle damage. Alternatively, a larger dorsiflexion

385 during the stance phase could be explained by a higher tendon strain, without affecting the SOL

386 strain itself. The muscle's F-L operating range and its interplay with the Achilles tendon function

387 depend on multiple factors, including tendon stiffness and a detailed understanding will require

388 further in vivo analyses to clarify the underlying mechanism.

390 Limitations 
391 Some limitations of the present study need to be acknowledged. First, in the measurement of $M_{p}$

392 used to calculate passive forces estimates, some participants displayed an inflection point (where

393 net dorsiflexion and plantarflexion moment converge on zero, Figure 1) slightly above or below

394 zero moment $(<1.5 \mathrm{Nm}$ or $\sim 7 \%$ of the peak passive moment). This can occur if the weight of the

395 leg transmits a small moment about the Biodex axis (i.e. small misalignment of ankle center of

396 rotation) or if the moment predicted from the weight of the foot has small errors. In these cases

397 the passive moment data was corrected for the offset. Second, the method used to calculate the

398 Achilles moment arm data (Manal et al., 2010), assumed the position of the ankle joint center

399 coincides with the marker placed on the medial malleolus. This could have resulted in a potential

400 misalignment of the ankle joint center, which might have affected the estimation of the moment

401 arm measurements. Investigations have also shown that muscle fascia structure can act as a

402 pathway for myofascial force transmission (Purslow et al. 2010), thus making more difficult to

403 completely isolate fascicle force production at single muscle level. Nevertheless, this factor has

404 been reported to be relatively small in intact muscles (Maas \& Sundercock, 2010) and most

405 likely did not significantly impacted our findings. Lastly, the heterogeneity of the CHF group

406 needs to be acknowledged as it might have influenced some of the findings. The difficulty

407 associated with enrolling large numbers of $\mathrm{CHF}$ participants to undertake prolonged

408 biomechanical tests prevented us from controlling for variables such as body mass, composition,

409 stature, or sex. Nevertheless, we tried to mitigate this problem by recruiting closely matched age

410 and physical activity-level control participants.

411 


\section{CONCLUSION}

413 This work suggests that a primary factor leading to lower passive forces in the SOL is likely a

414 reduction in muscle size. However, shorter muscle fascicles in CHF results in greater passive

415 forces for a given absolute muscle length, and might be linked to changes in CHF gait (Panizzolo

416 et al., 2014). Exercise that promotes calf muscle hypertrophy and serial sarcomerogenesis may

417 prove particularly beneficial in CHF patients.

418

419 ACKNOWLEDGEMENTS

420 The authors would like to acknowledge Ms. Kirsty McDonald and Ms. Maddison Jones for their

421 help during data collection, and all of the participants who volunteered for this study.

422

423

424 REFERENCES

425 Anker SD, Ponikowski PP, Clark AL, Leyva F, Rauchhaus M, Kemp M, Teixeira MM,

426 Hellewell PG, Hooper J, Poole-Wilson PA, Coats AJ. Cytokines and neurohormones relating

427 to body composition alterations in the wasting syndrome of chronic heart failure. Eur Heart $428 \quad J .1999 ; 20: 683-693$.

429

430 Arnold EM, Ward SR, Lieber RL, Delp SL. A model of the lower limb for analysis of human 431 movement. Ann Biomed Eng. 2010; 38: 269-279.

432

433

Azizi E, Roberts TJ. Muscle performance during frog jumping: influence of elasticity on 434 muscle operating lengths. Proc Biol Sci. 2010; 277: 1523-1530. 
436 Besier TF, Sturnieks DL, Alderson JA, Lloyd DG. Repeatability of gait data using a 437 functional hip joint centre and a mean helical knee axis. J Biomech. 2003; 36: 1159-1168.

439 Chua TP, Anker SD, Harrington D, Coats AJ. Inspiratory muscle strength is a determinant of 440 maximum oxygen consumption in chronic heart failure. Br Heart J. 1995; 74: 381-385.

441

442 Costa ML, Logan K, Heylings D, Donell ST, Tucker K. The effect of Achilles tendon 443 lengthening on ankle dorsiflexion: a cadaver study. Foot Ankle Int. 2006; 27: 414-417.

445 Delp SL, Anderson FC, Arnold AS, Loan P, Habib A, John CT, Guendelman E, Thelen DG. 446 OpenSim: open-source software to create and analyze dynamic simulations of movement. 447 IEEE Trans Biomed Eng. 2007; 54: 1940-1950.

449 Fülster S, Tacke M, Sandek A, Ebner N, Tschöpe C, Doehner W, Anker SD, von Haehling S. 450 Muscle wasting in patients with chronic heart failure: results from the studies investigating 451 co-morbidities aggravating heart failure (SICA-HF). Eur Heart J. 2013; 34: 512-519.

452

453 Geertsen SS, Kirk H, Lorentzen J, Jorsal M, Johansson CB, Nielsen JB. Impaired gait 454 function in adults with cerebral palsy is associated with reduced rapid force generation and 455 increased passive stiffness. Clin Neurophysiol. 2015; 126: 2320-2329. 456 
457 Gollapudi SK, Lin DC. Experimental determination of sarcomere force-length relationship in $458 \quad$ type-I human skeletal muscle fibers. J Biomech. 2009; 42: 2011-2016.

459

460 Godin G, Shepard RJ. A simple method to assess exercise behavior in the community. Can J $461 \quad$ Appl Sport Sci. 1985; 10:141-146.

462

463 Green DJ, Panizzolo FA, Lloyd DG, Rubenson J, Maiorana AJ. Soleus muscle as a surrogate 464 for health status in human heart failure. Exerc Sport Sci Rev. 2016; 44:45-50.

465

466 Harrington D, Anker SD, Chua TP, Webb-Peploe KM, Ponikowski PP, Poole-Wilson PA, 467 Coats AJ. Skeletal muscle function and its relation to exercise tolerance in chronic heart 468 failure. J Am Coll Cardiol. 1997; 30: 1758-1764.

469

470 Maas H, Sandercock TG. Force transmission between synergistic skeletal muscles through $471 \quad$ connective tissue linkages. J Biomed Biotechnol. 2010; Article ID 575672.

472

473 Maganaris CN, Baltzopoulos V, Sargeant AJ. Changes in Achilles tendon moment arm from 474 rest to maximum isometric plantarflexion: in vivo observations in man. J Physiol. 1998; 510: $475 \quad 977-985$.

476

477 Maganaris CN. Force-length characteristics of in vivo human skeletal muscle. Acta Physiol $478 \quad$ Scand. 2001; 172: 279-285. 
480 Magnusson G, Isberg B, Karlberg KE, Sylvén C. Skeletal muscle strength and endurance in 481 chronic congestive heart failure secondary to idiopathic dilated cardiomyopathy. $A m J$ $482 \quad$ Cardiol. 1994; 73: 307-309.

483

484 Maiorana A, O’Driscoll G, Cheetham C, Collis J, Goodman C, Rankin S, Taylor R, Green D. 485 Combined aerobic and resistance exercise training improves functional capacity and strength 486 in CHF. J Appl Physiol. 2000; 88: 1565-1570.

487

488 Manal K, Cowder JD, Buchanan TS. A hybrid method for computing achilles tendon 489 moment arm using ultrasound and motion analysis. J Appl Biomech. 2010; 26: 224-228.

490

491 Mancini DM, Walter G, Reichek N, Lenkinski R, McCully KK, Mullen JL, Wilson JR. 492 Contribution of skeletal muscle atrophy to exercise intolerance and altered muscle 493 metabolism in heart failure. Circulation. 1992; 85: 1364-1373.

494

495 McGowan CP, Kram R, Neptune RR. Modulation of leg muscle function in response to 496 altered demand for body support and forward propulsion during walking. J Biomech. 2009; $497 \quad 42: 850-856$.

499 Minotti JR, Pillay P, Oka R, Wells L, Christoph I, Massie BM. Skeletal muscle size: $500 \quad$ relationship to muscle function in heart failure. J Appl Physiol. 1993; 75: 373-381. 
502 Oskouei MA, Van Mazijk BC, Schuiling MH, Herzog W. Variability in the interpolated 503 twitch torque for maximal and submaximal voluntary contractions. J Appl Physiol. 2003; 95: $504 \quad 1648-1655$.

505

506 Panizzolo FA, Green DJ, Lloyd DG, Maiorana AJ, Rubenson J. Soleus fascicle length 507 changes are conserved between young and old adults at their preferred walking speed. Gait $508 \quad$ Posture. 2013; 38: 764-769.

509

510 Panizzolo FA, Maiorana AJ, Naylor LH, Dembo L, Lloyd DG, Green DJ, Rubenson J. Gait 511 analysis in chronic heart failure: the calf as a locus of impaired walking capacity. $J$ Biomech. $512 \quad 2014 ; 47: 3719-3725$.

513

514 Panizzolo FA, Maiorana AJ, Naylor LH, Lichtwark GA, Dembo L, Lloyd DG, Green DJ, 515 Rubenson J. Is the soleus a sentinel muscle for impaired exercise capacity in heart failure ? $516 \quad$ Med Sci Sport Exerc. 2015; 47: 498-508.

517

518 Pincivero DM, Green RC, Mark JD, Campy RM. Gender and muscle differences in EMG 519 amplitude and median frequency, and variability during maximal voluntary contractions of 520 the quadriceps femoris. J Electromyo Kines. 2000; 10: 189-196.

521

522 Rassier DE, MacIntosh BR, Herzog W. Length dependence of active force production in 523 skeletal muscle. J Appl Physiol. 1999; 86: 1445-1457. 
525 Purslow PP. Muscle fascia and force transmission. J Bodyw Mov Ther. 2010; 14:411-417.

526

527 Rubenson J, Pires NJ, Loi HO, Pinniger GJ, Shannon DG. On the ascent: the soleus operating

528 length is conserved to the ascending limb of the force-length curve across gait mechanics in 529 humans. J Exp Biol. 2012; 215: 3539-3551.

530

531 Silder A, Whittington B, Heiderscheit B, Thelen DG. Identification of passive elastic joint 532 moment-angle relationships in the lower extremity. J Biomech. 2007; 40: 2628-2635.

533

534 Sunnerhagen KS, Cider Å, Schaufelberger M, Hedberg M, Grimby G. 1998. Muscular 535 performance in heart failure. J Card Fail. 1998; 4: 97-104.

536

537 Tian M, Herbert RD, Hoang P, Gandevia SC, Bilston LE. Myofascial force transmission 538 between the human soleus and gastrocnemius muscles during passive knee motion. $J$ Appl $539 \quad$ Physiol. 2012; 113: 517-523.

541 Toth MJ, Ades PA, Tischler MD, Tracy RP, LeWinter MM. Immune activation is associated 542 with reduced skeletal muscle mass and physical function in chronic heart failure. Int $J$ $543 \quad$ Cardiol. 2006; 109: 179-187.

545 Toth MJ, Shaw AO, Miller MS, VanBuren P, LeWinter MM, Maughan DW, Ades PA. 546 Reduced knee extensor function in heart failure is not explained by inactivity. Int J Cardiol. $547 \quad 2010 ; 143: 276-282$. 
549 Van der Velden J. Diastolic myofilament dysfunction in the failing human heart. Pflugers $550 \quad$ Arch. 2011; 462: 155-163.

551

552 Van Hees HW, Ottenheijm CA, Granzier HL, Dekhuijzen PN, Heunks LM. Heart failure 553 decreases passive tension generation of rat diaphragm fibers. Int J Cardiol. 2010; 141: 275554283.

555

556 Volterrani M, Clark AL, Ludman PF, Swan JW, Adamopoulos S, Piepoli M, Coats AJ. 557 Predictors of exercise capacity in chronic heart failure. Eur Heart J. 1994; 15, 801-809.

558

559 Whittington B, Silder A, Heiderscheit B, Thelen DG. The contribution of passive-elastic 560 mechanisms to lower extremity joint kinetics during human walking. Gait Posture. 2008; 27, $561 \quad 628-634$.

562

563 Winters TM, Takahashi M, Lieber RL, Ward, S.R. Whole muscle length-tension 564 relationships are accurately modeled as scaled sarcomeres in rabbit hindlimb muscles. $J$ $565 \quad$ Biomech. 2011; 44: 109-115.

566

$567 \mathrm{Wu}$ Y. Changes in titin isoform expression in pacing-induced cardiac failure give rise to 568 increased passive muscle stiffness. Circulation. 2002; 106: 1384-1389. 


\section{Figure 1}

Net passive ankle joint moment-angle relationship for one representative participant.

The measured moment is displayed with solid black line, the 5th-order polynomial fit with dashed black line, the inflexion point where net dorsiflexion and plantarflexion moment converge on zero is displayed with a black circle $(\mathrm{O})$ 


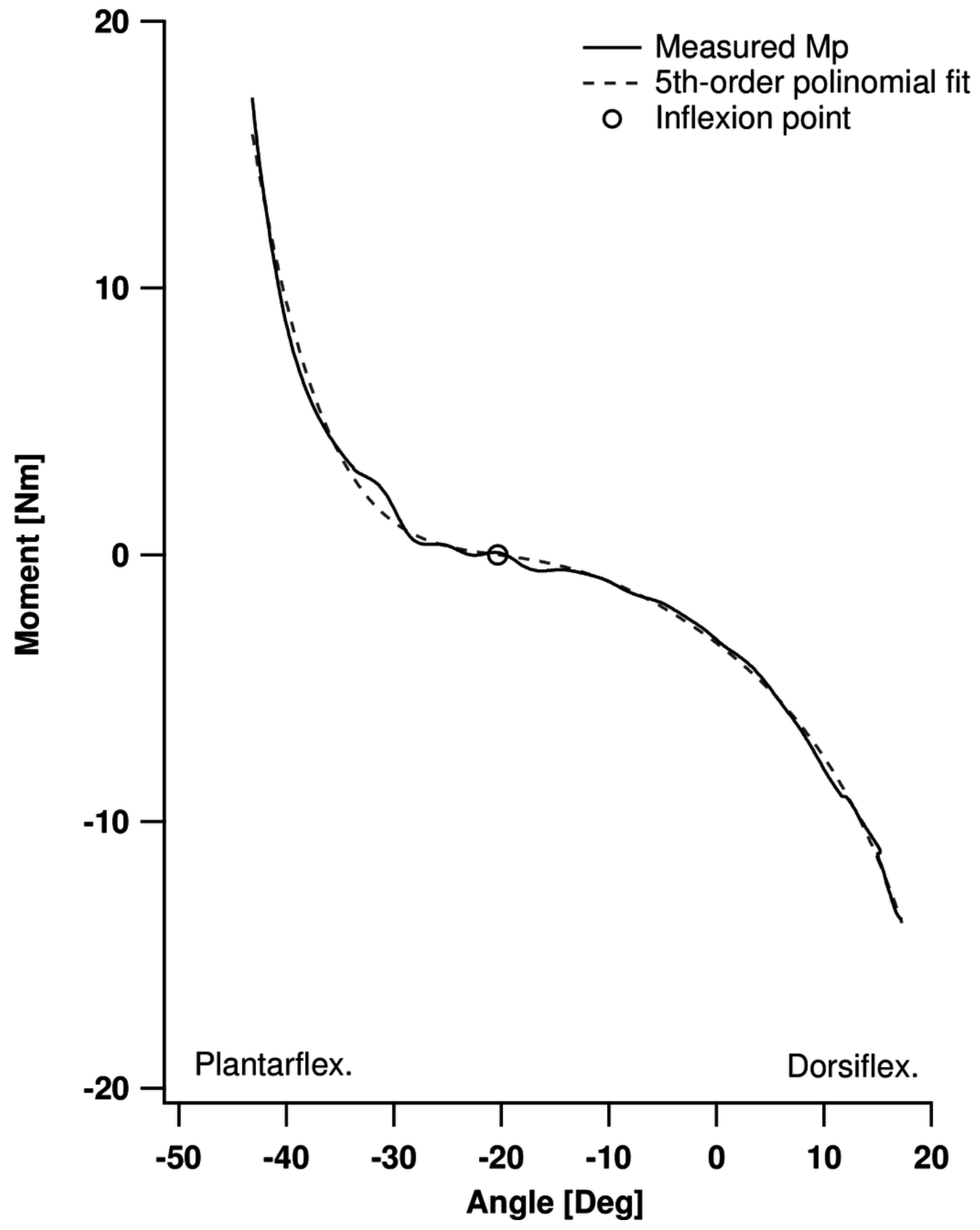


Figure 2

Soleus passive moment-angle relationship (evaluated at 0\%, 20\%, 40\%, 60\% and $100 \%$ of the angle's maximal dorsiflexion) (a) and passive moment-length relationship (b).

The chronic heart failure (CHF) group is displayed in grey triangles ( $\mathbf{\Lambda})$, and control group in black circles $(\mathbf{\bullet})$. Average curves are displayed \pm S.D. ${ }^{*}$ designates a statistical difference in passive moment between groups (ANOVA main effect; $p<0.05$; CHF vs. control)
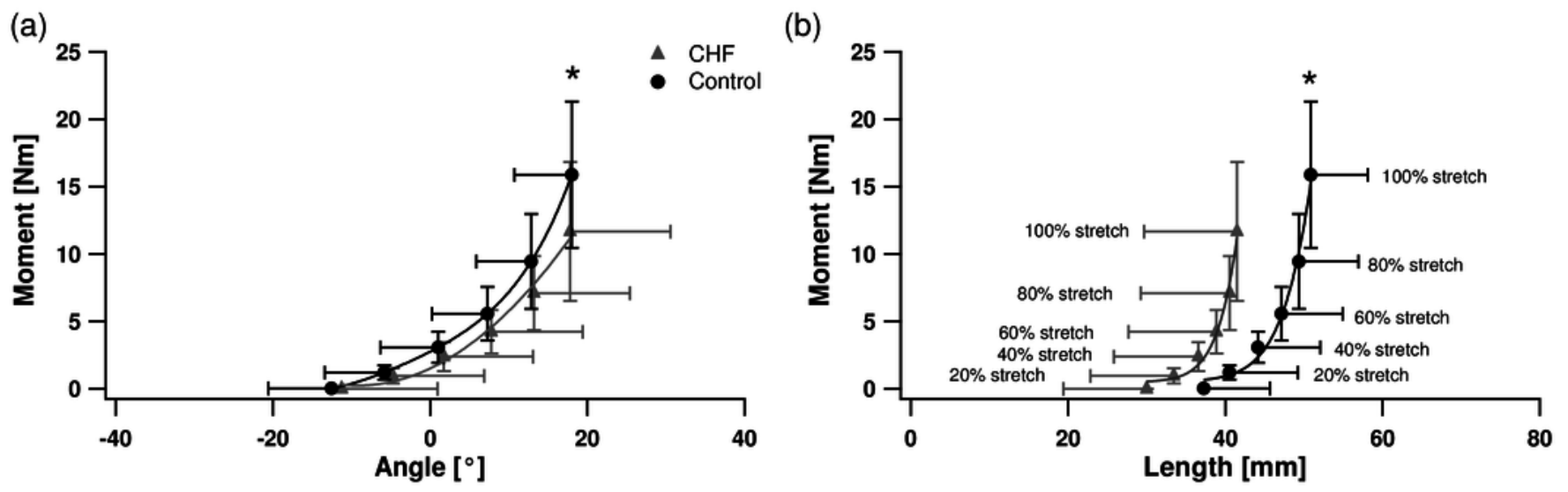
Figure 3

Soleus Passive force-length (F-L) relationship (a) and passive F-L relationship normalized by individual PCSA and $\mathrm{L}_{\text {slack }}(\mathrm{b})$.

Passive F-L is evaluated at $0 \%, 20 \%, 40 \%, 60 \%$ and $100 \%$ of the muscle's maximal stretch (a), passive F-L is evaluated at L_norm between 1.0 - 1.4 (b). The chronic heart failure (CHF) group is displayed in grey triangles ( $\mathbf{\Lambda})$, and control group in black circles ( $(\mathbf{0})$. Average curves are displayed \pm S.D. $*$ designates a statistical difference in passive force between groups (ANOVA main effect; $\mathrm{p}<0.05 ; \mathrm{CHF}$ vs. control). \# designates a significant difference in maximal passive fascicle length $\left(L_{\text {max }}\right)$ between groups $(p<0.05$; CHF vs. control)

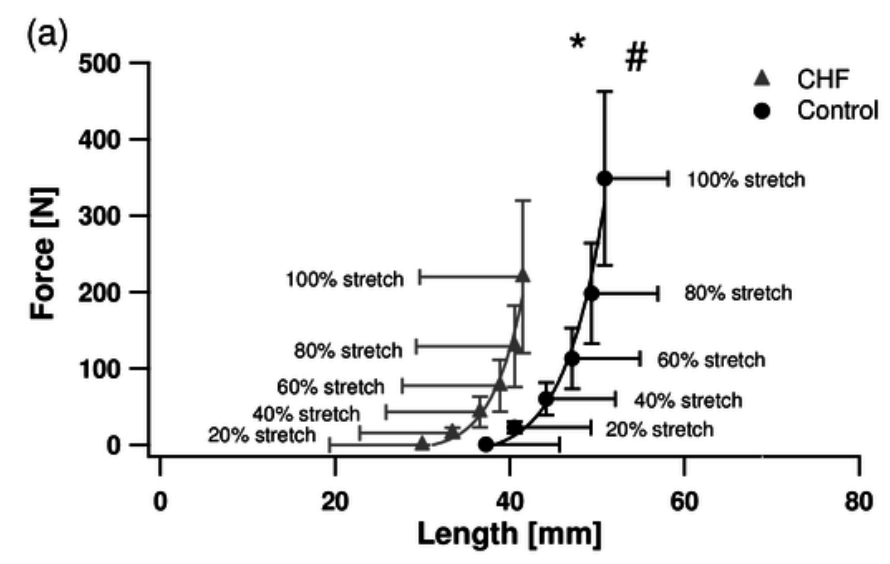

(b)

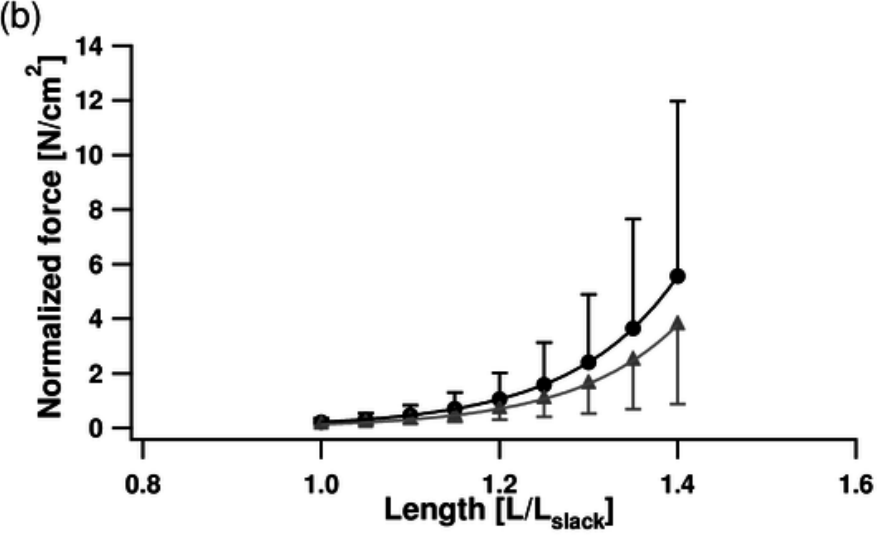




\section{Figure 4}

Normalized soleus peak voluntary force production and fascicle length.

Peak voluntary force production is normalized by the individual's maximal peak voluntary force production $\left(F_{\text {asol }}\right)$ while fascicle length is expressed in $\mathrm{mm}$. Data points (mean \pm S.D.) include measurements taken at joint angles greater and less than $10^{\circ}$ dorsiflexion. Muscle lengths were grouped in four clusters equally spaced along the fascicle length range. A data point at $\left(\mathrm{L}_{0}, 1\right)$ is included for reference purposes only. The chronic heart failure (CHF) group is displayed by grey triangles $(\boldsymbol{\Lambda})$, and control group by black circles $(\bullet)$

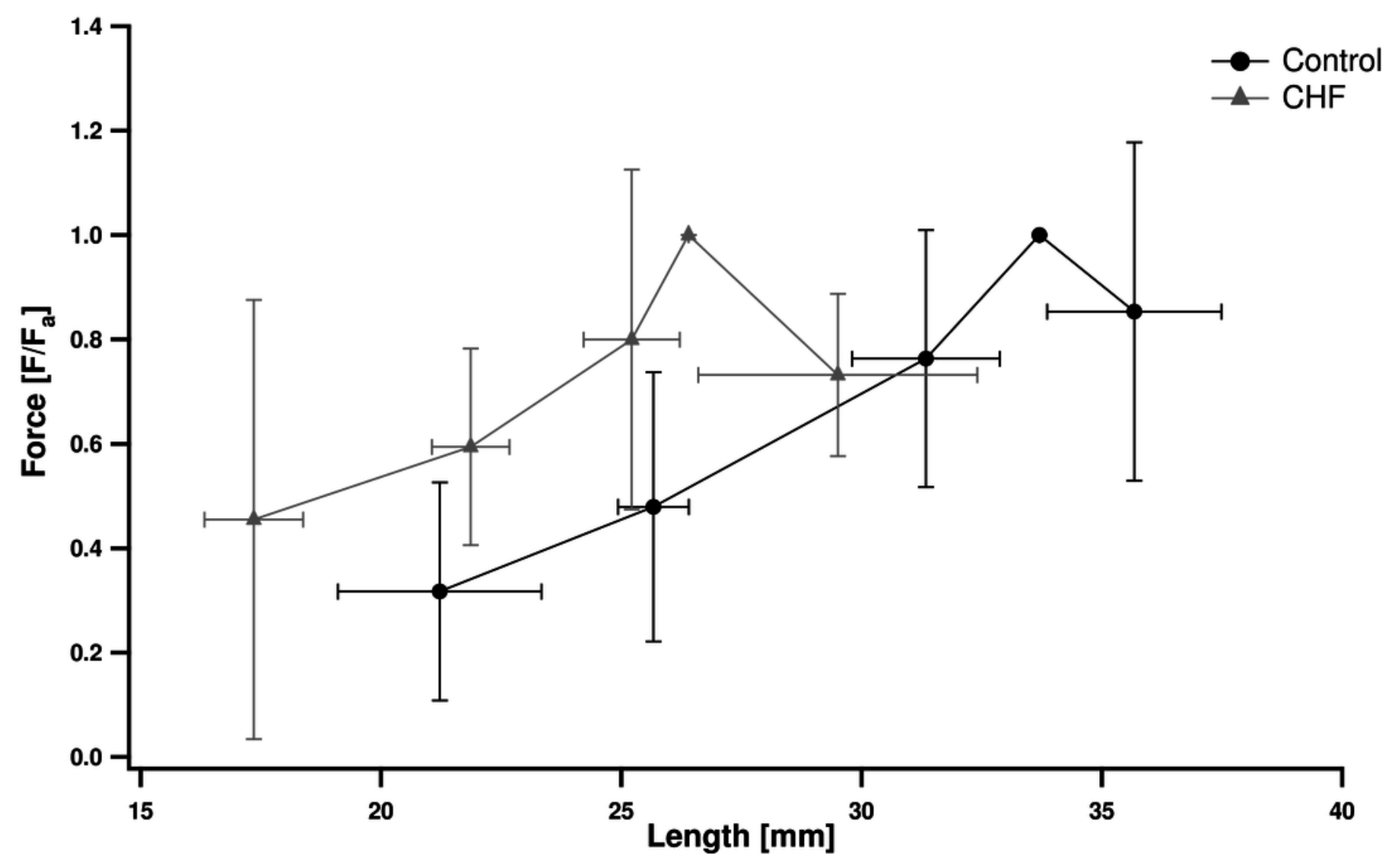




\section{Table $\mathbf{1}$ (on next page)}

Anthropometric characteristics of the participants involved in the study. Data are means \pm SD

Criteria for exclusion for the CHF group included severe renal (creatinine $>250 \mathrm{mmol} / \mathrm{l}$ or eGFR $<30 \mathrm{ml} / \mathrm{min} / 1.73 \mathrm{~m} 2$ ) or hepatic (bilirubin $>50 \mathrm{mmol} / \mathrm{l}$ ) dysfunction or unexplained anemia (hemoglobin $<100 \mathrm{~g} / \mathrm{l}$ ) or thrombocytopenia (platelets $<100 * 109 / \mathrm{l}$ ). Participants with the following contra-indications to exercise were excluded: unstable angina or exerciseinduced ischemia at low exercise levels (less than three metabolic equivalent units), severe aortic stenosis, severe mitral or aortic regurgitation, or hypertrophic cardiomyopathy 
1 Table 1

3

4

5

6

\begin{tabular}{cccc} 
Group & Age [yr] & Height $[\mathrm{cm}]$ & Weight $[\mathrm{kg}]$ \\
\hline Control & $62.7 \pm 5.6$ & $173.3 \pm 6.1$ & $69.7 \pm 8.5$ \\
CHF & $63.5 \pm 10.9$ & $168.2 \pm 9.6$ & $67.9 \pm 14.8$ \\
\hline
\end{tabular}

3

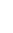
5 


\section{Table 2 (on next page)}

Muscle parameters. Data are means \pm SD

$*$ indicates a significant difference $(p<0.05)$ 


\section{$1 \quad$ Table 2}

\begin{tabular}{ccc} 
SOL muscle parameter & CHF & Control \\
\hline$L_{\text {slack }}[\mathrm{mm}]$ & $30.0 \pm 10.6$ & $37.3 \pm 8.4$ \\
$L_{\text {max }}[\mathrm{mm}]$ & $41.5 \pm 11.8^{*}$ & $50.9 \pm 7.3$ \\
$L_{\text {max }}-L_{\text {slack }}[\mathrm{mm}]$ & $11.5 \pm 5.2$ & $13.6 \pm 4.2$ \\
$L_{0}[\mathrm{~mm}]$ & $26.4 \pm 6.4^{*}$ & $33.7 \pm 8.4$ \\
Max strain $\left(L_{\text {norm }}\right)$ & $1.4 \pm 0.2$ & $1.4 \pm 0.2$ \\
PCSA $\left[\mathrm{cm}{ }^{2}\right]$ & $65.0 \pm 13.0^{*}$ & $91.0 \pm 20.5$ \\
Max $F_{p_{S O L}[\mathrm{~N}]}$ & $220.0 \pm 99.6^{*}$ & $348.6 \pm 113.4$ \\
$k_{1}[\mathrm{~N} / \mathrm{mm}]$ & $6.9 \pm 2.9$ & $12.0 \pm 13.8$ \\
$k_{2}[\mathrm{~N} / \mathrm{mm}]$ & $75.1 \pm 78.9$ & $82.6 \pm 85.7$ \\
$k_{1 \text { norm }}$ & $2.8 \pm 1.7$ & $4.1 \pm 4.1$ \\
$k_{2 \text { norm }}$ & $18.0 \pm 14.8$ & $26.3 \pm 32.2$ \\
& & \\
\end{tabular}

